\title{
Nueva propuesta para realizar una planificación microcurricular en el área de matemáticas
}

\section{New proposal to carry out a microcurricular planning in the mathematics area}

Juan Fernando Barrazueta Samaniego

Fabián Eugenio Bravo Guerrero

César Augusto Trelles Zambrano

Universidad de Cuenca, Ecuador

Autor para correspondencia: juanfer023@hotmail.com, fabianbravo@yahoo.com, cesar.trellesz@ucuenca.edu.ec

Fecha de recepción: 1 de Julio de 2018 - Fecha de aceptación: 12 de Septiembre de 2018

Resumen: Parte fundamental del proceso enseñanza-aprendizaje es la planificación, pues permite al docente guiar el aprendizaje de sus estudiantes, identificando los objetivos, destrezas y criterios de evaluación planteados en el currículo que van a ser abordados según el tema tratado, también permite indicar cuales son las estrategias metodológicas a ser aplicadas, los recursos a ser utilizados en el desarrollo del tema, los indicadores de logros de aprendizaje, es decir, aspectos que permiten identificar si los estudiantes adquirieron los conocimientos mínimos requeridos y las técnicas o instrumentos a ser utilizados para evaluar el nuevo conocimiento. Dentro de las estrategias metodológicas o actividades de aprendizaje es en donde se proponen la aplicación de cinco estrategias, que han mejorado los resultados en la enseñanza-aprendizaje de la matemática; dichas estrategias son: relación, experimentación, aplicación, cooperación y la transferencia, más conocidas por sus siglas como la REACT. La implementación de éstas dentro de la planificación marca la diferencia entre aulas tradicionalistas y constructivistas, siendo las últimas las que mejores resultados presentan dentro de la educación matemática.

Palabras clave: destrezas; criterios de evaluación; estrategias metodológicas; indicadores de logros; REACT

Abstract: An important part of the teaching- learning process is the planning. It helps teachers to guide their students due to the educator is able to identify the objectives, skills and evaluation criteria which were proposed in the curriculum and will be addressed according to the subject studied. In addition, the planning shows the best methodological strategies that will be applied, the resources that will be used during the performance of the topic, and the indicators for the performance criteria; it means, the aspects that allow to identify if the students acquired the minimum knowledge that are required and the techniques or instruments to be used in order to evaluate the new knowledge. In the methodological strategies or learning activities space is where five interesting strategies are suggested which have improved the results in the teaching-learning process of the mathematic. These strategies are: the relation, experimentation, application, cooperation and the transference, all of them known as REACT. The implementation of all these strategies in a planning makes the difference between a traditional and constructivist class, being the last one which have the best results in the mathematical education.

Key Words: strategies; evaluation criteria; methodological strategies; indicators of the performance criteria; REACT 


\section{Introducción}

Las dificultades que se presentan en el proceso de enseñanza-aprendizaje, específicamente en el área de matemáticas, pueden deberse a múltiples situaciones didácticas como adidácticas, sin embargo, el directamente responsable de dicho proceso sigue siendo el docente, pues dentro del aula de clase es el encargado de guiar el aprendizaje por parte de sus estudiantes y en él está la decisión de continuar aplicando métodos o estrategias tradicionalistas (conductistas) o por el contrario, implementar acciones creativas, novedosas y atractivas en las que los estudiantes participen y se involucren de una manera activa en su aprendizaje (constructivismo).

El presente artículo pretende ser un apoyo a la labor docente, pues presenta como algo novedoso en la planificación, la utilización de cinco estrategias metodológicas, que se pueden utilizar en el desarrollo de cualquier tema de matemática planteado en el currículo y que han dado grandes resultados dentro de la educación matemática (National commission on mathematics and science teaching for the 21 st century, 2000), por parte de los estudiantes que han sido partícipes de este proceso.

\section{Definición}

Dentro de cualquier contexto en el que se desarrolle el ser humano, al momento de realizar una actividad ya sea de índole educativo, deportivo, económico, entre otras, existe un proceso que resulta imprescindible para llegar a la consecución de las metas u objetivos planteados al inicio de dicha actividad, a este proceso se lo conoce como planificación; pero ¿qué es planificar? o ¿qué representa una planificación?; antes de responder las preguntas planteadas, se ponen a consideración ciertas generalidades que guardan estrecha relación con el proceso antes mencionado, como por ejemplo:

- Es prospectiva (orientada hacia el futuro).

- Tiene objetivos (hacia dónde va).

- Requiere uso de recursos.

- Contiene actividades.

- Se basa en procedimientos.

Todos estos aspectos permiten visualizar a la planificación como un proceso cuyo punto de partida son los objetivos de la organización, mismos que promueven el uso de recursos materiales y humanos, a través del cumplimiento de ciertas actividades propuestas. También se la puede considerar como una acción que consiste en utilizar un conjunto de procedimientos organizados en actividades y acciones entrelazadas entre sí, las cuales son previstas con anticipación y que tienen como misión influir en el curso de diferentes acontecimientos, con el fin de alcanzar una situación deseable, mediante el uso eficiente de medios y recursos limitados. "En consecuencia, tanto los alumnos como los maestros experimentarán mucha menos frustración si se asigna un tiempo adecuado para la asimilación y la integración del conocimiento" (Baroody, 2005) 
Dentro del campo educativo la planificación resulta ser una actividad esencial, pues es la directamente responsable de que el proceso enseñanza aprendizaje resulte exitoso. Al respecto el Instructivo para planificaciones curriculares para el sistema nacional de educación, señala que:

La planificación permite organizar y conducir los procesos de enseñanza y aprendizaje necesarios para la consecución de los objetivos educativos. Además, lleva a reflexionar y tomar decisiones oportunas, pertinentes, tener claro qué necesidades de aprendizaje poseen los estudiantes, qué se debe llevar al aula y cómo se puede organizar las estrategias metodológicas, proyectos y procesos para que el aprendizaje sea adquirido por todos, y de esta manera dar atención a la diversidad de estudiantes (Ministerio de Educación, 2016)

Continuando en el ámbito educativo, que es la esencia de este trabajo, a continuación se presentan los objetivos de la planificación.

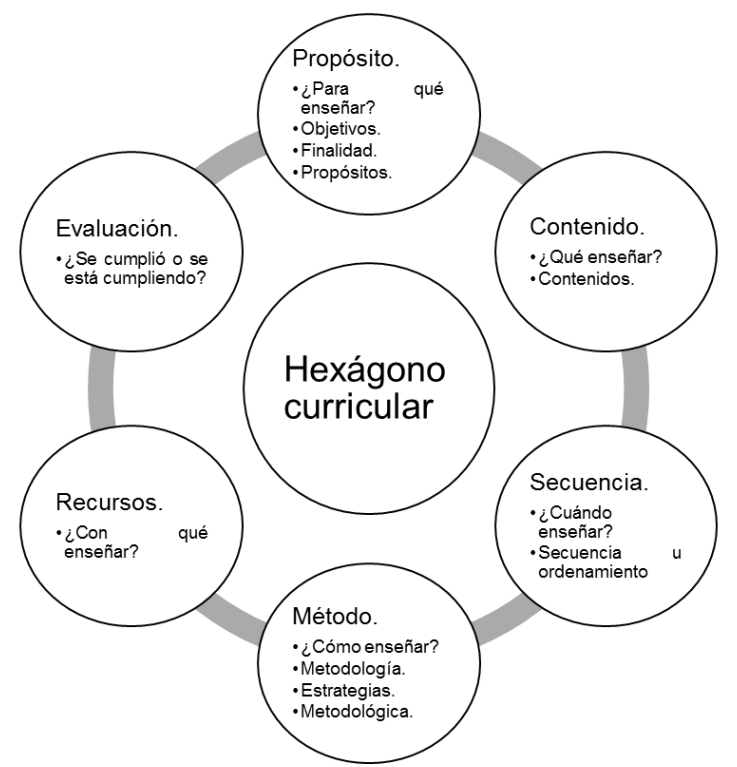

Figura 1. Hexágono curricular.

Fuente. Propia

\section{Clases de Planificación}

La planificación educativa tiene diferentes niveles de concreción curricular, mismos que se ponen a consideración.

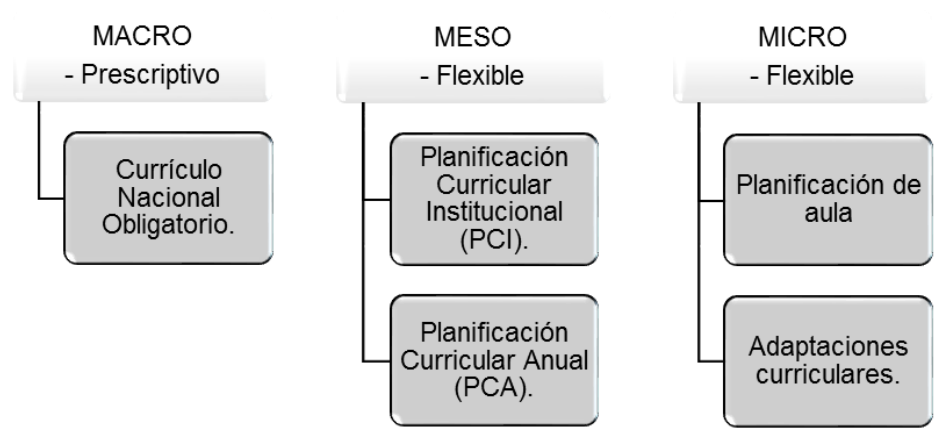

Figura 2. Clases de Planificación. - Fuente. Propia. 
Planificación macrocurricular. Está elaborada por expertos dentro del ámbito educativo, pedagogo y curricular, es aquí donde se definen: el perfil, los objetivos, las destrezas con criterio de desempeño, los indicadores de evaluación, mismos que constan dentro del Currículo Nacional Obligatorio. Cabe mencionar que dicho currículo resulta ser flexible pues las instituciones educativas son quienes van a definir los contenidos que van a abordar, en función de los requerimiento de sus estudiantes y de las necesidades de la institución y de la sociedad. (Ministerio de Educación, 2016)

Planificación mesocurricular. Se fundamenta en la planificación macrocurricular, es decir, en el currículo obligatorio, las autoridades y docentes de cada institución educativa resultan ser los directamente responsables de su elaboración (PCI, PCA), respondiendo siempre a las especificidades de su contexto. (Ministerio de Educación, 2016)

Planificación microcurricular. Se fundamentan en el PCI y PCA de cada institución, cada docente resulta ser el responsable de su elaboración y lo hace en función de las necesidades de sus estudiantes. Esta planificación se la realiza por cada año de Educación General Básica (EGB) y Bachillerato General Unificado (BGU). (Ministerio de Educación, 2016)

\section{Estructura Curricular}

\section{Enfoque Pedagógico}

La Actualización y fortalecimiento curricular de la educación general básica aplicada en el año 2010 se basa en diferentes concepciones teóricas y metodológicas del quehacer educativo; como por ejemplo considerar algunos principios de la Pedagogía Crítica, insertada en diferentes estructuras metodológicas, en las que predominan las vías cognitivistas y constructivistas (Actualización y fortalecimiento curricular de la educación general básica 2010, 2010). Lo que busca este enfoque metodológico es la implementación de procesos de enseñanza-aprendizaje activos, dinámicos y participativos que generen en los estudiantes habilidades y destrezas, para que éstos a su vez sean capaces de aplicarlos en la construcción de sus propios procedimientos para llegar a la solución efectiva de cualquier situación problémica dentro y fuera del contexto educativo. También busca una formación integral basada en una práctica continua de valores como: la justicia, solidaridad, responsabilidad, cuidado del medio ambiente y la responsabilidad social. Una de las asignaturas, que por su naturaleza, constituye un gran aporte a la formación integral es la Matemática, en la que intervienen tres factores dentro de su actividad pedagógica:

Los primeros tienen que ver con los individuos y los mecanismos mentales que intervienen en el pensamiento matemático de cada persona. Los segundos son las estructuras matemáticas y su propio dinamismo. Los terceros son las relaciones de las estructuras con la realidad, en particular con las aplicaciones (Castro \& Castro, 2014)

\section{Elementos Curriculares}

Todo currículo responde a las preguntas: ¿para qué enseñar?, ¿qué enseñar?, ¿cuándo enseñar?, ¿cómo enseñar?, ¿qué, cómo y cuándo evaluar?; mismas que se corresponden con los fines, objetivos, contenidos, metodología, recursos y evaluación; elementos esenciales a la hora 
de realizar cualquier tipo de planificación curricular (Instructivo: Planificaciones curriculares para el sistema nacional de educación, 2016)

Respecto a las preguntas antes mencionadas, Barcia explica y específica ¿a qué hace referencia cada una de ellas?

¿Qué hay que enseñar? En este componente se incluyen los objetivos, contenidos. Los contenidos deben entenderse en sentido amplio, incluyendo hechos, conceptos, procedimientos o habilidades, actitudes, valores, normas, etc. ¿Cuándo hay que enseñar? Cómo ordenar y secuenciar los objetivos y contenidos. ¿Cómo hay que enseñar? Una vez explicitado lo que se debe enseñar y cuándo, hay que referirse a cómo hacerlo para facilitar al máximo la consecución de los objetivos (Rodríguez \& Barcia, 2011)

\section{Fines}

Se basa en el perfil de salida de cada uno de los niveles y subniveles de educación y son desempeños que debe cumplir el estudiante con un grado de generalización de destrezas y conocimientos específicos. Este desempeño se refleja a través de las destrezas de mayor generalización "saber hacer", de conocimiento "saber" y de valores humanos "ser".

\section{Objetivos}

Buscan el desempeño integral del estudiante, de manera específica en cada una de las distintas áreas de estudio planteados en el sistema educativo. Respondiendo siempre a las preguntas:

- ¿Qué acción o acciones de alta generalización deben realizar los estudiantes?

- ¿Qué debe saber? Conocimientos asociados y cuáles son los logros de desempeño esperados.

- ¿Para qué? Contextualización en el entorno natural y social. (Instructivo: Planificaciones curriculares para el sistema nacional de educación, 2016)

\section{Contenidos}

Representa el "saber hacer", a través del dominio de las destrezas con criterio de desempeño o de las competencias planteadas en el currículo; con una o varias acciones que desarrollan los estudiantes asociadas a un conocimiento teórico y basadas a diferentes niveles de complejidad que caracterizan los criterios de desempeño. 


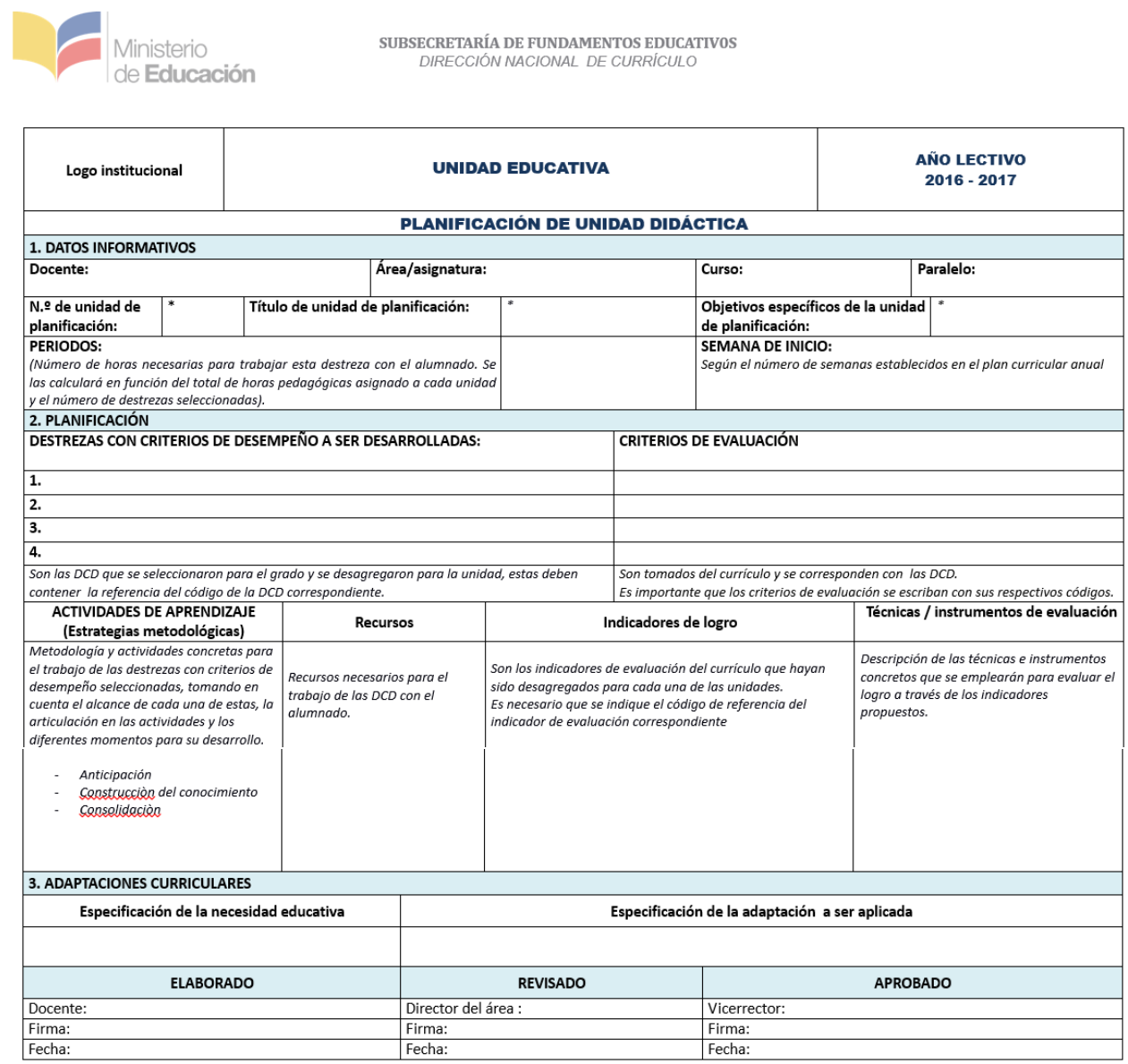

Figura 3. Planificación Microcurricular Fuente. Ministerio de Educación.

\section{Metodología}

La metodología debe estar en función de las capacidades, intereses, actitudes y respuestas del grupo. Además, es preciso diversificar los tiempos y los modelos e instrumentos de evaluación y las actividades planificadas deben respetar los diferentes estilos de aprendizaje y las necesidades educativas de los alumnos/as (Rodríguez \& Barcia, 2011)

Están constituidas por orientaciones metodológicas y didácticas que amplían la información expresada por las destrezas con criterio de desempeño o las competencias, a la vez que ofrecen sugerencias para desarrollar de una forma efectiva diversos métodos y técnicas que conducen al desarrollo de éstas dentro y fuera del aula de clase. Son procesos que guían el desempeño de los docentes con sus estudiantes que buscan generar un ambiente agradable y propicio para que se desarrolle el aprendizaje, fortaleciendo en la formación de valores y de hábitos de estudio.

\section{Recursos}

Se pueden considerar como recursos toda clase de material que lleva por objetivo facilitar el proceso de enseñanza aprendizaje, mismo que según su utilización pueden ser o no didáctico. 
Por ejemplo "Un vídeo para aprender qué son los volcanes y su dinámica será un material didáctico (pretende enseñar), en cambio un vídeo con un reportaje del National Geographic sobre los volcanes del mundo a pesar de que pueda utilizarse como recurso educativo, no es en sí mismo un material didáctico (sólo pretende informar)" (Fernandez, 2015)

Son todos aquellos elementos o materiales que los docentes utilizan en el desarrollo de su clase para facilitar el aprendizaje de sus estudiantes, dichos materiales pueden ser: digitales, bibliográficos, concretos, audio visuales, entre otros.

\section{Evaluación}

La actividad evaluativa es inherente a los procesos educacionales, empero vale cuestionarnos sobre ¿cómo entendemos este proceso?, ¿cómo lo llevamos a la práctica en nuestro accionar diario?, ¿evaluamos porque el sistema nos lo exige? o ¿evaluamos para mejorar continuamente el proceso educativo?, ¿evaluamos apegados a un marco teórico conceptual? o ¿entendemos la evaluación como el simple hecho de tomar pruebas y asignar calificaciones?, ¿evaluamos permanentemente el aprendizaje de nuestros estudiantes? o ¿evaluamos simplemente al término de una unidad, bloque, capítulo o ciclo académico?, las respuestas que los docentes den a las anteriores preguntas, sin duda reflejan la concepción que tienen sobre educación (Trelles, Bravo, \& Barrazueta, 2017)

"Al referirnos a la evaluación educativa es importante contemplarla en su totalidad, como un proceso dinámico y sistemático y ubicarla como integral y fundamental de toda acción educativa" (Pezo, 1995). La evaluación está dada a través de lineamientos y promoción que respondan al modelo pedagógico de cada institución y que a su vez estén relacionados con la normativa nacional. Son evidencias concretas de los resultados de aprendizaje, precisan el desempeño esencial que deben demostrar los estudiantes. Es importante que se incluyan procesos de autoevaluación y coevaluación entre los estudiantes.

\section{Elementos Esenciales para Elaborar la Planificación Didáctica}

\section{Datos Informativos}

Son los encargados de contextualizar la planificación de acuerdo al nombre del docente, grado o curso, paralelo, el área o asignatura, al tema, el tiempo que se requiere para desarrollar las tareas de aprendizaje, especificando el número de horas clase a ser utilizadas e indicando las semanas que va a emplear, mismas que deben guardar relación con lo expuesto en la planificación curricular anual (PCA).

También se encuentran aspectos como:

\section{Título de la Unidad de Planificación}

Que en la mayoría de los casos lo dispone cada una de las instituciones y guardan estrecha relación con su misión y visión.

Objetivos Educativos Específicos 
Buscan la consecución del desempeño integral de los estudiantes en cada área y año de estudio. Responden a las preguntas:

- ¿Qué acción o acciones deben realizar los estudiantes?

- ¿Qué deben saber?

- ¿Para qué, deben saber?

\section{Planificación}

El docente luego de explicitar los datos informativos, plantea actividades de enseñanza aprendizaje que buscan generar el desarrollo y dominio de las destrezas con criterio de desempeño, así como los recursos didácticos a ser utilizados y actividades que permitan evidenciar la consecución de los indicadores de evaluación.

A continuación se presenta una breve explicación de cada uno de los elementos que integra la planificación:

\section{Destrezas con Criterio de Desempeño a ser Desarrolladas}

Se relacionan con el "saber hacer", es decir, el conjunto de acciones a ser desarrolladas por el estudiante en las que se conjuguen un conocimiento teórico con un determinado nivel de complejidad de los criterios de desempeño. Responden a las siguientes preguntas:

- ¿Qué deben saber hacer?

- ¿Qué deben saber?

- ¿Con qué grado de complejidad?

\section{Indicadores Esenciales de Evaluación}

Son los requisitos mínimos del dominio de destrezas con criterio de desempeño por parte de los estudiantes. Responden a las siguientes preguntas:

- ¿Qué deben saber, entender y hacer los estudiantes?

- ¿Cuáles son los conocimientos esenciales del año?, es decir que grado de complejidad debe dominar.

- ¿Qué resultados evidencian el aprendizaje?, es decir que actitudes deben mostrar los estudiantes.

El docente también podrá implementar otros indicadores de evaluación en caso de ser requeridos.

\section{Actividades de Aprendizaje (Estrategias Metodológicas)}

Son procesos metodológicos que generan el desarrollo de las destrezas con criterio de desempeño, es decir actividades concretas y específicas, buscando siempre que éstas se encuentren bien articuladas, dentro del proceso de enseñanza aprendizaje.

\section{Recursos}


Resulta necesario detallar todos los recursos a ser utilizados, esto demuestra que han sido seleccionados con anterioridad asegurando su pertinencia.

\section{Indicadores de Logro}

Representan los indicadores de evaluación propuestos en el currículo, y que para incluirlos en las planificaciones por unidades, necesariamente deben estar desagregados y responder a los temas planteados en dicha planificación.

\section{Técnica (Instrumentos de Evaluación)}

Son evidencias que permiten apreciar y validar el aprendizaje, las herramientas a ser utilizadas deben ser bien detalladas y concisas, ya que facilitarán el proceso de evaluación.

\section{Adaptaciones Curriculares}

Este campo será utilizado en caso de que se presenten algún o algunos estudiantes con necesidades educativas específicas, se tendrá que especificar el tipo de adaptación a ser aplicada y los lineamientos que se establecieron previamente en el PCI. Y por último se presentan datos de los nombres de la persona que elaboró la planificación, de quien revisó y aprobó dicha planificación, también se incluyen las firmas de responsabilidad y en las fechas en las que se realizaron dichos procesos.

\section{Destrezas y Habilidades}

Las destrezas representan el "saber hacer", es decir, la capacidad de dominar y aplicar el conocimiento adquirido en la resolución de diversas situaciones problémicas dentro de su cotidianeidad.

El dominio de las destrezas, requieren de la interiorización de los conocimientos, procedimientos, el desarrollo de la capacidad crítica y creativa. El perfeccionamiento y afianzamiento de éstas resulta ser un proceso gradual, en el que intervienen diferentes tipos de actividades cognitivas y experiencias.

Las destrezas se enseñan y se aprenden dentro del contexto en el cual se desarrolla el estudiante, en dichas situaciones es en donde éste le encuentra sentido a lo que está aprendiendo, y más aún cuando puede apreciar la aplicación del nuevo conocimiento en la resolución de alguna situación problémica didáctica o adidáctica que durante algún tiempo venía intentando resolverla. La aplicación de una destreza permite al estudiante empezar a dominar los conocimientos de una manera individualizada.

El "saber hacer" implica el dominio, reflexión y aplicación de lo aprendido en varias actividades cotidianas, poniendo en manifiesto la significancia y relevancia de los nuevos conocimientos aprendidos planteados en el currículo.

\section{Aprendizaje de las Destrezas}

\section{Realización de Acciones}


Como ya se explicó, el aprendizaje de las destrezas trata sobre el "saber hacer", es decir la puesta en práctica de los aprendizajes, lo que se consigue a través de la realización de diferentes acciones ordenadas y finalizadas; sin embrago existen todavía en el proceso de aprendizaje, textos y maestros que conducen a los estudiantes a memorizar conceptos y acciones, que lo único que hacen es mecanizar y crear conductas repetitivas de procesos, por ejemplo; cuando se trabaja el tema de operaciones combinadas con supresión de signos, no basta con realizar un ejercicio, pues, no todos los ejercicios que se presentan o proponen tienen la misma estructura, lo que indica que todos los ejercicios se resuelven de distinta forma, aunque ciertas reglas se mantengan como por ejemplo el orden en el cual se deben suprimir los signos de agrupación; lo que pone en manifiesto que el dominio de las destrezas se consigue solo a base de resolver todas las acciones propuestas.

\section{Ejercitación}

En la actualidad se propende a dosificar las tareas dentro y fuera del aula de clase, pero esto no quita, que se tengan que realizar tantos ejercicios como sean necesarios para que cada estudiante pueda dominar las acciones y conceptos que éstos implican.

La ejercitación bien dirigida, permite al docente tener idea del nivel y ritmo de aprendizaje de sus estudiantes, de las dificultades que presentan al momento de resolver los ejercicios planteados y de buscar las posibles soluciones, todos estos aspectos le permiten especificar en su planificación el tipo y la cantidad de actividades que va a proponer a los estudiantes, para que éstos tengan la posibilidad de poder consolidar su aprendizaje.

1. Reflexión sobre la propia actividad.

Luego de realizar una debida ejercitación, viene un proceso mucho más complejo que permite a los estudiantes consolidar su aprendizaje, este proceso es la reflexión sobre las actividades que está realizando y cuáles son las condiciones propicias en las que pueden ser aplicadas. Si se quiere dominar las destrezas planteadas no basta solo con la resolución de ejercicios y el aprendizaje de conceptos, resulta necesario adquirir los nuevos conocimientos de una forma reflexiva que genera el análisis de los actos y como éstos pueden ser mejorados.

2. Aplicación en diferentes contextos.

Los nuevos contenidos aprendidos formarán parte de un aprendizaje efectivo cuando los estudiantes vean la aplicabilidad e éstos no solo en situaciones previsibles, como por ejemplo en la resolución de diferentes tipos de ejercicios, si no en diferentes contextos, lo que obliga, a tener una gran diversidad de ejercicios que permitan observar de una manera clara y oportuna la aplicabilidad de los contenidos siempre que éstos sean requeridos.

\section{La enseñanza de las destrezas}

Para lograr la enseñanza de las destrezas se deben dar el cumplimiento de las siguientes condiciones.

1. Partir de situaciones significativas y funcionales.

Para que el aprendizaje pueda ser dado de una manera efectiva, se debe dar sentido a los contenidos intentando mostrar la conveniencia de su utilidad, es decir, demostrar a los 
estudiantes para qué sirve y cuál es su función; así sea como base para obtener un nuevo conocimiento. Intentando responder de esta a manera a las clásicas preguntas de los estudiantes como: ¿para qué, me sirve aprender esto? y ¿cuándo voy a utilizar?

2. Progreso y orden.

En búsqueda de un aprendizaje efectivo, es necesario que absolutamente todas las actividades a ser realizadas estén debidamente planificadas es decir que guarden un orden específico y atiendan a un proceso gradual de complejidad.

3. Presentación de modelos.

Resulta necesario evidenciar modelos de cómo deben presentarse los contenidos que son objeto de aprendizaje, en ellos se presentan algoritmos con el desarrollo de todo el proceso, que presente una visión completa de las distintas fases y acciones que la componen.

4. Práctica guiada y ayudas de distinto grado.

El avance y progreso de la asignatura, estará determinado en gran medida por las características que presentan los diferentes recursos que se irán presentando a lo largo del proceso de aprendizaje de nuevos contenidos.

5. El trabajo independiente.

Parte importante del proceso de aprendizaje, es que el estudiante tenga la posibilidad de realizar actividades procedimentales de forma independiente.

\section{Enseñanza contextual}

Las evaluaciones realizadas por el ministerio de Educación del Ecuador en los años 1996 y 2007 pruebas “Aprendo", dieron como resultados promedios de 7,93/20 y 6,56/20 respectivamente, en el área de matemática. En el año 2008 el Ministerio de Educación del Ecuador genera un nuevo instrumento de evaluación denominado "Ser Ecuador", en el cuál el área de matemática tiene una puntuación de 7,86/20. A más de obtener estos datos de forma cuantitativa, también se pudo recabar información sobre las razones por la cuáles los docentes justificaban el cumplimiento o incumplimiento de los contenidos y objetivos de la reforma, la queja de siempre: la desarticulación que existía entre los niveles educativos es decir la educación superior culpaba a la educación secundaria y ésta a la educación primaria del bajo rendimiento de los estudiantes; la inexactitud en cuanto a los temas y destrezas a ser impartidas dentro del aula de clase y de la poca información con respecto a los criterios e indicadores de evaluación a ser aplicados.

Todos estos datos sin duda generaron la preocupación en el Sistema Educativo Ecuatoriano, razón por la cual surgió la necesidad de buscar soluciones, una de éstas es el "Plan Decenal de Educación 2006-2015" que se aprobó en noviembre del 2006, que tuvo como política mejorar la calidad de educación y que fue renovado con ciertas modificaciones en 2016.

A pesar de todos los cambios generados e implementados, todavía se pueden notar algunas falencias, que son muestra de que no se está haciendo el buen trabajo que se debería hacer o que se pudiera hacer para que los estudiantes puedan asimilar, interiorizar, aplicar y 
hacer suyos los conocimientos impartidos dentro del aula de clase. Al no dominar estos aspectos, los estudiantes se están quedando relegados en cuanto a la excelencia en ésta área del conocimiento y de las asignaturas afines.

Los métodos y técnicas utilizados en el proceso de aprendizaje suelen ser muy tradicionalistas, como por ejemplo, las típicas clases magistrales en las que el docente era el expositor y responsable del ritmo de la clase, mientras que el educando tomaba una actitud pasiva como un mero receptor de la información; este método sin duda alguna funcionó y dio buenos resultados en el pasado y para los educandos de aquella "época", pero se puede notar que en la actualidad este método no está generando los resultados esperados, por lo que se necesita de manera urgente cambiar los métodos y estrategias de aprendizaje, las cuáles deben ser implementadas en donde se efectúa el proceso de aprendizaje, es decir en el aula.

Si el cambio está dentro del aula, el principal responsable sin duda alguna es el docente, él es quien debe cambiar esa visión tradicionalista de intentar abarcar todos los contenidos propuestos en el currículum educativo y el de asignar una valoración cuantitativa luego de una evaluación realizada, lo cual está muy lejos de conseguir estudiantes excelentes en el área de matemática.

El docente ha tenido un papel fundamental en el proceso formativo: el guiar la clase, aplicando su dominio de los contenidos, además de la habilidad para transmitirlos. Pero esta no es la realidad del docente de las matemáticas; un alto porcentaje de profesores de matemáticas no tiene formación pedagógica; y peor aún, en otros casos tienen escasa preparación disciplinar en matemáticas (Bravo, Trelles, \& Barrazueta, 2017)

El objetivo principal de la educación en el área de matemática debe ser el generar un entendimiento profundo de los conceptos fundamentales del programa de estudio. Una de las estrategias que ayudan a generar este entendimiento se la conoce como la "Enseñanza Contextual", que se basa en aprender a entender y cómo los profesores enseñan a entender.

La corriente pedagógica que permite desarrollar el Enseñanza Contextual es el "Constructivismo", que en esencia promulga: "En el sentido más general, el modo contemporáneo de aprendizaje es que la persona construye nuevo conocimiento y entendimiento basándose en lo que ya conoce y cree” (Bransford, Brown, \& Cocking, 1999, pág. 25)

Ampliando lo expuesto anteriormente, Robinson Castro y Rubby Castro proponen que: El aprendiz construye su propia realidad o al menos la interpreta de acuerdo a la percepción derivada de su propia experiencia, de tal manera que el conocimiento de la persona es una función de sus experiencias previas, estructuras mentales y las creencias que utiliza para interpretar objetos y eventos (Castro \& Castro, 2014)

La mayoría de investigadores que se pronuncian acerca del constructivismo coinciden en que el estudiante aprende mejor, cuando su conocimiento es adquirido a través de la exploración y de un aprendizaje activo, en el que el estudiante toma un rol protagónico en su aprendizaje a través de diferentes prácticas que pongan a prueba su ingenio y en el que tengan que explicar su razonamiento en cuanto a la resolución de situaciones problémicas, estas actividades permiten 
ver a los estudiantes la relación que existe entre los diferentes temas en vez de plantearlos y exponerlos como temas aislados.

La diferencia entre una clase tradicional y una constructivista consiste en que en esta última los estudiantes presentan una predisposición para involucrarse de manera directa con su aprendizaje y compartir con sus compañeros las diferentes estrategias que le permiten efectuar la resolución correcta de cualquier actividad, en vez de esperar a recibirlas por parte del docente. Los beneficios de implementar esta aula constructivista son múltiples, por ejemplo: se fomenta el aprendizaje cooperativo en el que lo estudiantes comparten sus inquietudes y posibles soluciones, ampliando de esta manera su visión con respecto al tema tratado; los estudiantes se involucran de forma directa en las diferentes actividades prácticas y en las aulas se genera un ambiente de confianza, interés y necesidad de aprender.

Consecuentemente la visión constructivista del conocimiento es concebir los procesos cognitivos como construcciones eminentemente activas del sujeto que conoce, en interacción con su ambiente físico y social. Aplicando a la pedagogía, es un referente que guía la acción (Maldonado, 2000)

La implementación de éstas actividades marca la diferencia entre los docentes que triunfan con aquellos estudiantes que muchos otros docentes se han dado por vencidos y son quienes implementando estrategias novedosas en su ejercicio docente influyen y marcan de una forma positiva la vida de sus estudiantes. Muchas de estas actividades son implementadas por parte de los docentes dentro del proceso de aprendizaje de una manera empírica, las preguntas serían: ¿Con qué frecuencia las aplico? y ¿Soy consciente, de la importancia y utilidad de estas actividades?, las respuestas a estas preguntas marcarán la diferencia entre las aulas tradicionalistas y las aulas constructivistas.

Múltiples son los métodos y estrategias que se aplican en las aulas constructivistas, pero todas éstas coinciden en cinco estrategias que son las más utilizadas por los docentes, mismas que son conocidas como "Estrategias de Enseñanza Contextual" (National commission on mathematics and science teaching for the 21 st century, 2000), que se resumen en cinco palabras: Relación, Experimentación, Aplicación, Cooperación y Trasferencia, que se abrevian con las siglas REACT, que representan las estrategias con las cuales los estudiantes aprenden mejor y guardan estrecha relación con el aprendizaje en el contexto del estudiante que es un principio fundamental del constructivismo.

A continuación se presentará una descripción de cada una de las estrategias enunciadas y de materiales didácticos que validan cada una de éstas.

\section{Relación}

Esta estrategia se basa en el constructivismo pues es una de las más poderosas, consiste en contextualizar el aprendizaje, es decir que el estudiante active su conocimiento previo a través de las experiencias que ha tenido anteriormente. 
Esta estrategia para activar lo que el estudiante conoce sobre el tema que va a ser abordado y para que luego pueda "conectar" éstos con un nuevo conocimiento, cuando este proceso se lo realiza con éxito el entendimiento por parte de los estudiantes es casi inmediato. Causando en los estudiantes una reacción de asombro al conseguir la resolución exitosa de un ejercicio o problema en el que ha venido trabajando durante cierto tiempo y más aún al ver la aplicabilidad de lo aprendido. El entendimiento hará que el estudiante le encuentre sentido a los nuevos contenidos impartidos dentro del aula de clase.

El docente es el único responsable de planificar diferentes situaciones que faciliten un aprendizaje efectivo por parte de los estudiantes, pues muchas de la veces éstos no conectan con éxito la nueva información con la ya existente y es aquí donde el docente debe generar ambientes propicios para que los estudiantes puedan activar sus conocimientos previos, reconozcan la importancia y la relevancia de hacerlo, lo que facilitará la conexión con los nuevos conocimientos, aplicando de esta manera la relación.

Un aspecto importante es reconocer que le aprendizaje de alguna idea o concepto matemático por parte de los estudiantes no es un proceso terminal, sino que se robustece en función de una serie de conexiones que los mismos estudiantes van logrando en sus experiencias de aprendizaje (Santos, 2014)

Por ejemplo, al tratar el tema de poliedros que pertenece al bloque de geometría, el docente que imparte una clase tradicionalista comienza con la definición seguido de un ejemplo que por lo general lo toma del texto guía, es decir:

Definición: "Poliedro es una figura que tiene sus caras planas y están limitadas por polígonos" (Matemática 7, 2016) y "llámese polígono una porción de un plano limitada por segmentos de rectas" (Wentworth \& Smith, 2010)

Ejemplo:
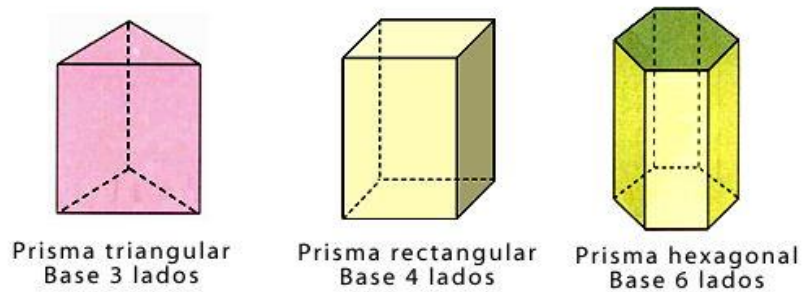

Figura 2. Prismas

Fuente: https://www.portaleducativo.net/quinto-basico/524/Prismas-y-piramides

Éste es un claro ejemplo de una clase típica de algunos docentes tradicionalistas, el docente que utiliza la estrategia de "relación", comienza sus clases haciendo preguntas a sus estudiantes, en las que se pongan en manifiesto las experiencias de éstos, por ejemplo: ¿Qué figuras pueden apreciar en los objetos que están en el aula de clase? o ¿Qué forma tienen los objetos que traen en su mochilas?, con las respuestas dadas por los estudiantes el docente afianzará el conocimiento previo de éstos a través de la obtención de un sin número de nombres de figuras o formas que ellos aprecian. 
El niño trabaja de forma manipulativa para identificar diferencias, similitudes y, por supuesto, conocer el nombre de cada uno de los cuerpos o sólidos geométricos, pues es en la etapa sensorial cuando se representa todo lo que nos rodea. Para ello son importantes la observación y la relación de los elementos, en una palabra: abrir el espíritu de observación (Zorrilla, 2013)

Luego de que el docente realiza esta actividad, los estudiantes pueden entender la importancia de los conocimientos previos, pues sentirán que resulta familiar y accesible aprender, interiorizar y dominar el concepto de poliedro, pues esta estrategia les permitirá relacionarla con cualquier objeto que esté a su alrededor. Es muy importante que los estudiantes puedan conectar los nuevos conocimientos con lo que ellos ya saben o dominan y de lo cual ya están convencidos.

La aplicación de esta estrategia permite al docente tomar como punto de partida de la construcción del nuevo conocimiento, el conocimiento previo que posee el estudiante sobre el tema a ser tratado; ahora la pregunta es: ¿Cómo el docente puede saber lo que el estudiante conoce previamente? Esta pregunta se la puede responder a través de diferentes métodos que permiten recolectar información de parte de los estudiantes:

- Experiencia. Representan las actividades que tienen características similares realizadas por los docentes y estudiantes o entre docentes.

- Investigación. Representa una actividad debidamente documentada realizada por los estudiantes, en las que se evidencian las ideas que tienen los estudiantes.

- Sondeo. Representan preguntas o actividades planificadas cuidadosamente cuyo objetivo es proporcionar información sobre los conocimientos o convicciones que tienen los estudiantes.

Todas estas actividades permiten obtener datos de los conocimientos previos de los estudiantes, que sin duda alguna van a ser los cimientos sobre los cuales se pueden empezar a construir los nuevos aprendizajes; ahora, estos conocimientos previos también pueden dificultar el aprendizaje, cuando éstos son erróneos o equivocados, es aquí donde se ve la necesidad de aplicar una técnica, como por ejemplo la resolución de ejercicios en los que se pueda visualizar el error en la conceptualización que maneja el estudiante, es decir, a través de una demostración cuidadosa que permita destruir esas concepciones erróneas, para luego empezar a construir concepciones que sean perfectamente razonables y comprensibles.

\section{Experimentación}

La experimentación consiste en aprender a través de la experimentación, descubrimiento e invención, es decir "aprender haciendo", basadas en la realización de diferentes actividades como:

- Actividades manipulativas.

- Actividades de resolución de problemas.

- Actividades de laboratorio.

Actividades manipulativas. Son actividades en las que los estudiantes a través del contacto con el material concreto, pueden modelar y comprender concepciones abstractas, por 
ejemplo al trabajar con poliedros, sería muy útil utilizar "material concreto", que permite al estudiante reconocer los elementos y principales características de los poliedros. Otro método que se puede utilizar es la ayuda de un softwares educativos como Cabri, Geogebra, Matlab, entre otros; que si bien no representa un material concreto, sí representa un material manipulativo que permite realizar cambios sobre la marcha y observar de forma inmediata dichos cambios en la gráfica, respondiendo siempre a la pregunta ¿Qué pasaría si......? Todo este tipo de actividades bien planificadas mejoran el desempeño del estudiante.

Actividades de Resolución de Problemas. Son acciones orientadas a despertar la creatividad en el estudiante, pues desarrollan el pensamiento, generan una visión crítica ante la problemática y mejora la comunicación dentro del grupo de estudio, también ayuda a mejorar el aprendizaje de conceptos y definiciones del tema tratado. Estas actividades son las que permiten al estudiante entender la importancia del aprendizaje del nuevo conocimiento, al momento de ver su aplicación en la resolución de un problema, hecho que da sentido al aprendizaje.

Como ejemplo, se va a continuar analizando el tema de poliedros. Después de introducir en concepto a través de la estrategia de la relación expuesta anteriormente, el docente puede plantear la siguiente problemática ¿Qué forma debe tener una caja, si en ella deseo guardar un balón?, los estudiantes trabajando de forma individual o grupal, buscarán la forma de resolver la problemática planteada, aquí lo interesante es la forma en que los estudiantes van a proponer sus posibles soluciones, luego se llegará a consensuar con todo el grupo sobre la mejor manera de resolver el problema.

La realización exitosa de esta actividad motiva a que el estudiante sienta la importancia, la necesidad de conocer y de utilizar los poliedros. Esta actividad va guiada por el docente quien es el responsable de facilitar el análisis, definir los enfoques y las conclusiones finales, demostrar las conceptualizaciones en el momento que se requieran.

Actividades de Laboratorio. Es una actividad mucho más compleja que las anteriores, pues requiere de una mayor planificación, aquí los estudiantes trabajan en grupos tomando datos, analizando estos, sacan conclusiones, hacen predicciones y reflexionan sobre el fundamento teórico del fenómeno estudiado.

Muchas de las veces los estudiantes se ven inmersos en una práctica de laboratorio dentro de una clase de matemática, sin percatarse de ello. Pues se puede pedir que se analicen la forma del aula dentro de su respectivo grupo, luego que compartan esta información con el resto de grupos y representen estos datos a través de un cuadro y de un gráfico. Esta actividad permite a los estudiantes generar nuevas formas de representar la información obtenida.

Al momento en que los estudiantes utilizan la información propia de su entorno, permite una mejor predisposición de éstos para analizar fenómenos a través de la utilización de diferentes modelos matemáticos que les permiten mejorar el entendimiento de la parte conceptual.

Todos estos métodos están orientados a que los estudiantes generen su propio conocimiento a través de la realización de diferentes actividades debidamente planificadas, ahora 
bien, esto no implica que el docente no pueda brindar información de forma directa a sus estudiantes.

La aplicación de clases $100 \%$ magistrales predispone el control total del ambiente de aprendizaje por parte del docente y por el contrario el aprendizaje por descubrimiento determina un control de la clase por parte del estudiante, en cualquiera de los dos casos no representa una forma eficaz para generar un buen aprendizaje, por ello es conveniente buscar un punto intermedio en el que se fusionen estos dos tipos de aprendizaje basados en la relación y la experimentación que son las estrategias responsables de crear un nuevo conocimiento.

\section{Aplicación}

Determina el aprendizaje de nuevos conocimientos a través de la puesta en práctica de éstos, es decir, cuando los estudiantes realizan su propia conceptualización. La aplicación necesita de una mayor atención por parte del docente pues es el encargado de generar y proponer a los estudiantes ejercicios y problemas que sean reales y relevantes, es decir, situaciones con las que los estudiantes se puedan encontrar en su cotidianeidad, lo que sin duda va a motivar a éstos para buscar las posibles soluciones para dichas situaciones, dando importancia y utilidad a los nuevos conocimientos adquiridos.

Esta estrategia es la encargada de responder la "pregunta del millón” ¿Para qué me sirve aprender esto? Por ejemplo en una clase de geometría se aborda el tema de poliedros y se propone el siguiente problema:

Determine el área de la base y de las caras laterales de la Pirámide de Guiza ubicada en Egipto, cuyas medidas son:

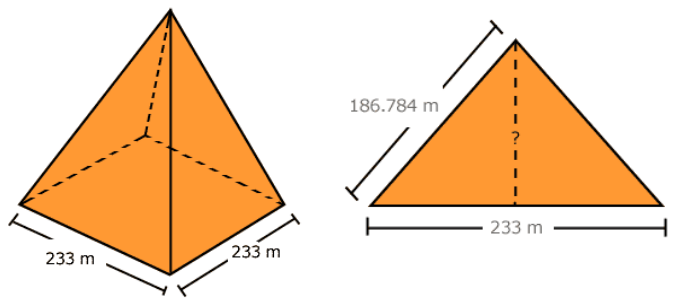

Figura 3. Medidas de la pirámide de Guiza. Fuente: cursosinea.conevyt.org.mx

El propósito del ejercicio planteado es que los estudiantes identifiquen, relacionen las características de la pirámide y utilicen las fórmulas para determinar el área de la base de forma cuadrada y de las caras laterales que tiene forma triangular.

Aquí los estudiantes pueden darse cuenta de la importancia de la parte conceptual de los nuevos conocimientos y de la aplicabilidad en el ámbito de la arquitectura y del diseño, ahora, no a todos los estudiantes les gusta o entienden sobre dichas áreas, es por ello que durante todo el transcurso del año es recomendable el buscar problemas para tratar diversas situaciones con las que se pueda encontrar el estudiante. Es imposible esperar tener resultados diferentes o nuevas formas de resolver un problema, cuando se siguen proponiendo siempre actividades y problemas 
tradicionales que lo único que buscan es la aplicación de una fórmula y mediante esta obtener una respuesta predecible.

La labor del docente resulta ser fundamental pues es el encargado de direccionar el aprendizaje y centrarse en los aspectos fundamentales de los nuevos conocimientos, luego tiene que planificar tareas, actividades que sean novedosas, realistas y que puedan desafiar el intelecto de sus estudiantes, considerando el nivel educativo en el que están sus estudiantes. Las actividades a ser propuestas deben estar bien planificadas pues corren el riesgo de que si son muy sencillas el estudiante puede llegar a pensar que domina por completo el conocimiento, lo que puede generar un desinterés por aprender, pues el estudiante puede verse desmotivado. Por el contrario si las actividades son demasiado complejas el estudiante podrá llegar a pensar que adquirir ese nuevo conocimiento es imposible, no podrá progresar en su entendimiento y menos generar un aprendizaje efectivo. Por ello es recomendable el proponer actividades en las cuales los estudiantes puedan apreciar el avance en la construcción de su conocimiento y el reforzamiento de éstos.

El objetivo principal de la aplicación es generar en los estudiantes una actitud positiva frente al aprendizaje de nuevos conocimientos a través de una comprensión profunda basada en la aplicabilidad de lo aprendido, lo cual le da una razón para aprender, es decir, se crea una necesidad de conocer y aprender.

\section{Cooperación}

La cooperación consiste en aprender a través de compartir e interactuar, pues cuando los estudiantes trabajan en grupo pueden resolver diversas situaciones complejas con poca ayuda externa al grupo de trabajo.

Cuando los estudiantes trabajan en grupo existe una mayor predisposición por parte de ellos para aprender ya que no se sentirán intimidados o avergonzados al momento de realizar una pregunta por más simple que esta parezca y de la misma forma podrán compartir con el resto del grupo lo mucho o poco que hayan aprendido, utilizando un lenguaje muy propio del grupo lo que sin duda generará un mejor entendimiento de los nuevos conocimientos. Esta actividad permite generar valores como el respeto a las diferentes opiniones que surgen de los integrantes del grupo, pues frente a un problema cada integrante propondrá diferentes formas de resolverlo y luego en conjunto consensuarán la mejor forma de resolverlo de forma correcta. La realización exitosa del problema conlleva una automotivación y mayor seguridad, por parte de cada uno de los estudiantes al momento de resolver un problema de forma individual.

Al momento de trabajar en grupo se debe ser muy cuidadoso, ya que se deben asignar roles a cada integrante del grupo, esto genera una actitud de responsabilidad y da importancia a la actividad realizada por cada miembro del grupo, pues solo al finalizar la actividad propuesta se darán cuenta que el éxito depende de todos y cada uno de los integrantes del grupo. Al momento de trabajar en grupos y de no definir los roles de cada integrante, pueden surgir dificultades en el proceso de aprendizaje, ya que existirán integrantes que no deseen participar o por el contrario, integrantes que manipulen el proceso del aprendizaje cooperativo, por ello, es necesario que 
exista una dependencia de la orientación docente, para la conformación de grupos y la realización de las actividades propuestas.

Al respecto David Johnson y Roger Johnson ${ }^{1}$ (1990); líderes en el aprendizaje cooperativo, proponen ciertas pautas que permiten crear ambientes propicios para que los estudiantes puedan aprender e interiorizar los nuevos conocimientos evitando el surgimiento de situaciones negativas dentro del proceso de aprendizaje. Al respecto se pueden considerar las siguientes pautas:

- "Estructurar interdependencia positiva dentro de los grupos. Johnson y Johnson proponen que hay que hacer que los estudiantes sientan que van a tener éxito si y solo si el grupo tienen éxito, es decir, que los integrantes del grupo tengan objetivos y recompensas comunes, asignando tareas de forma equitativa y haciendo que los integrantes se vuelvan fuente de información entre sí. (National commission on mathematics and science teaching for the 21 st century, 2000).

- Hacer que los estudiantes interactúen mientras hacen sus tareas y asegurarse que esa interacción proviene de trabajar en las mismas. Consiste en promover la interacción en la resolución de las tareas y el estimular a los estudiantes a que propongan y compartan con el resto del grupo sus inquietudes y posibles soluciones frente a la realización de una actividad. (National commission on mathematics and science teaching for the 21 st century, 2000).

- Hacer que todos los estudiantes sean responsables individualmente por la ejecución de las tareas y que no se apoyen en el trabajo de los otros. Johnson y Johnson al respecto proponen dos estrategias para que los estudiantes sean responsables de sus actividades: a) evaluar de forma individual y no grupal, b) tomar aleatoriamente el trabajo de un estudiante en representación de todos los miembros del grupo. (National commission on mathematics and science teaching for the 21 st century, 2000).

- Hacer que los alumnos aprendan a usar habilidades interpersonales y de trabajo en grupos pequeños. Consiste en desarrollar habilidades que ayuden a mejorar el trabajo en grupo, como por ejemplo: liderazgo, toma de decisiones, fomentar la confianza y el manejo de conflictos. (National commission on mathematics and science teaching for the 21 st century, 2000).

- Asegurar que los grupos analicen cómo están desempeñando sus funciones. Resulta necesario realizar una evaluación sobre el cumplimiento de los roles que están desempeñando cada uno de los estudiantes dentro de su grupo, pues esto ayudará de cierta forma a que reflexionen sobre su trabajo y que luego lo puedan desempeñar de mejor manera" (National commission on mathematics and science teaching for the 21 st century, 2000).

La realización de un aprendizaje cooperativo requiere de una mayor labor por parte del docente, pues es el responsable de formar los grupos de trabajo, ser un guía y observador de la resolución de las actividades propuestas, esto le permitirá reconocer las dificultades y proponer las posibles soluciones sobre la marcha, es decir, que el docente no va a ser solo un mero expositor, también tendrá que ser un facilitador y un observador. Como se puede apreciar el

\footnotetext{
${ }^{1}$ Johnson David, and Johnson Roger. "Using Cooperative Learning in Mathematics", Cooperative Learning in Mathematics: A Handbook for Teachers, Neal Davidson, ed. Menlo Park, CA: Innovative Learning, AddisonWesley. 1990.
} 
trabajo cooperativo no es fácil de llevarlo a cabo, pero bien vale la pena el sacrificio, considerando que esta actividad conlleva un mayor entendimiento de los nuevos conocimientos a través un trabajo constante y efectivo.

\section{Transferencia}

La transferencia consiste en aprender en el contexto de la aplicación de los nuevos conocimientos en nuevas situaciones, es decir proponer problemas del contexto de los estudiantes en los que se pueda apreciar la aplicación del nuevo conocimiento adquirido.

Cuando las clases de matemáticas se llevan de una manera tradicional, es decir una clase en la que se proponen ejercicios habituales, en los que se evidencian la memorización de una fórmula y la aplicación de ésta para resolverlos, lo que hacen es mecanizar el proceso de aprendizaje, volviéndolo monótono y generando desmotivación en los estudiantes. Por el contrario la transferencia invita a que se propongan nuevas situaciones que puedan despertar la curiosidad y el interés de los estudiantes creando una motivación intrínseca por aprender en cada uno de ellos.

Como por ejemplo en el campo de la geometría se puede tomar como referencia sus propios hogares, pues se les puede pedir que describan la forma que tiene cada una de las habitaciones y la cantidad de objetos o cosas que caben en cada uno de ellos, se puede inferir que la mayoría de los estudiantes reaccionarán y responderán de forma emocional a ser un tema con el cuál se encuentran plenamente identificados, el tema por su naturaleza da la posibilidad de que se puedan realizar diferentes actividades como por ejemplo. Un debate, un análisis matemático de la representación de sus hogares. Se puede analizar si la toma de datos es correcta y más aún si su representación de forma gráfica coincide con los valores de los datos tomados, también se puede realizar una encuesta con los estudiantes dentro del aula de clase en primera instancia y luego con toda la institución educativa, abordando el mismo tema, analizar los datos obtenidos y representarlos a través de una tabla de datos y de forma gráfica, en la cual se expliquen los resultados obtenidos sobre las opiniones de los estudiantes.

Este tipo de ejercicios son los que generan esa curiosidad y motivación por aprender, involucrando a los estudiantes de forma directa con su proceso de aprendizaje, pues van a sentir la importancia de conocer y aplicar lo aprendido en algún tema con el cual se encuentren plenamente identificados. El aprendizaje basado en la Relación, Experimentación, Aplicación Cooperación y Transferencia, genera un aprendizaje significativo basado en la creatividad y la satisfacción del estudiante al momento de aprender.

\section{Bibliografía}

Baroody, A. (2005). El pensamiento matemático de los niños. Madrid: Machado libros.

Bransford, J., Brown, A. L., \& Cocking, R. R. (1999). How people learn: Brain, mind experience and school. National Academy Press, 10. 
Bravo, F., Trelles, C., \& Barrazueta, J. (2017). Reflexiones sobre la evolución de la clase de matemáticas en el bachillerato ecuatoriano. INNOVA Research Journal, 1-12.

Castro, R., \& Castro, R. (2014). Álgebra desde una perspectiva didáctica. Bogota: Ecoe ediciones.

Ecuador, M. d. (2010). Actualización y fortalecimiento curricular de la educación general básica 2010. Quito: Don Bosco.

Ecuador, M. d. (2016). Matemática 7. Quito: Don Bosco.

Educación, M. d. (2016). Instructivo: Planificaciones curriculares para el sistema nacional de educación. Quito: Don Bosco. Obtenido de Ministerio de Educación.

Educación, M. d. (Agosto de 2016). Ministerio de Educación. Obtenido de Ministerio de Educación: https://educacion.gob.ec/

Fernandez, F. (14 de Junio de 2015). Los medios didácticos y los recursos educativos. Santiago de Chile, Chile, Chile.

Maldonado, M. E. (2000). Teorías psicológicas del aprendizaje. Cuenca: U de Cuenca.

National commission on mathematics and science teaching for the 21 st century. (2000). Before It's Too Late: A report to the nation from the National commission on mathematics and science teachingfor the 21 stcentury. En Jessup (Ed.). (pág. 4). Education publications center.

Pezo, E. (1995). Evaluación Educativa. Propad.

Rodríguez, M., \& Barcia, M. (2011). Didáctica y Currículum para el desarrollo profesional docente. Madrid: Dykinson.

Santos, M. (2014). La resolución de problemas matemáticos. Fundamentos cognitivos. México: Trillas.

Trelles, C., Bravo, F., \& Barrazueta, J. (2017). ¿Cómo evaluar los aprendizajes en matemáticas? INNOVA Research Journal, 35-51.

Wentworth, J., \& Smith, D. (2010). Geometría plana y del espacio. Boston: Ginn y compañía.

Zorrilla, C. (2013). Didáctica de las Matemáticas. La progresión de las matemáticas de preescolar a secundaria. México: Trillas. 


\section{Anexo A. Modelo de planificación}

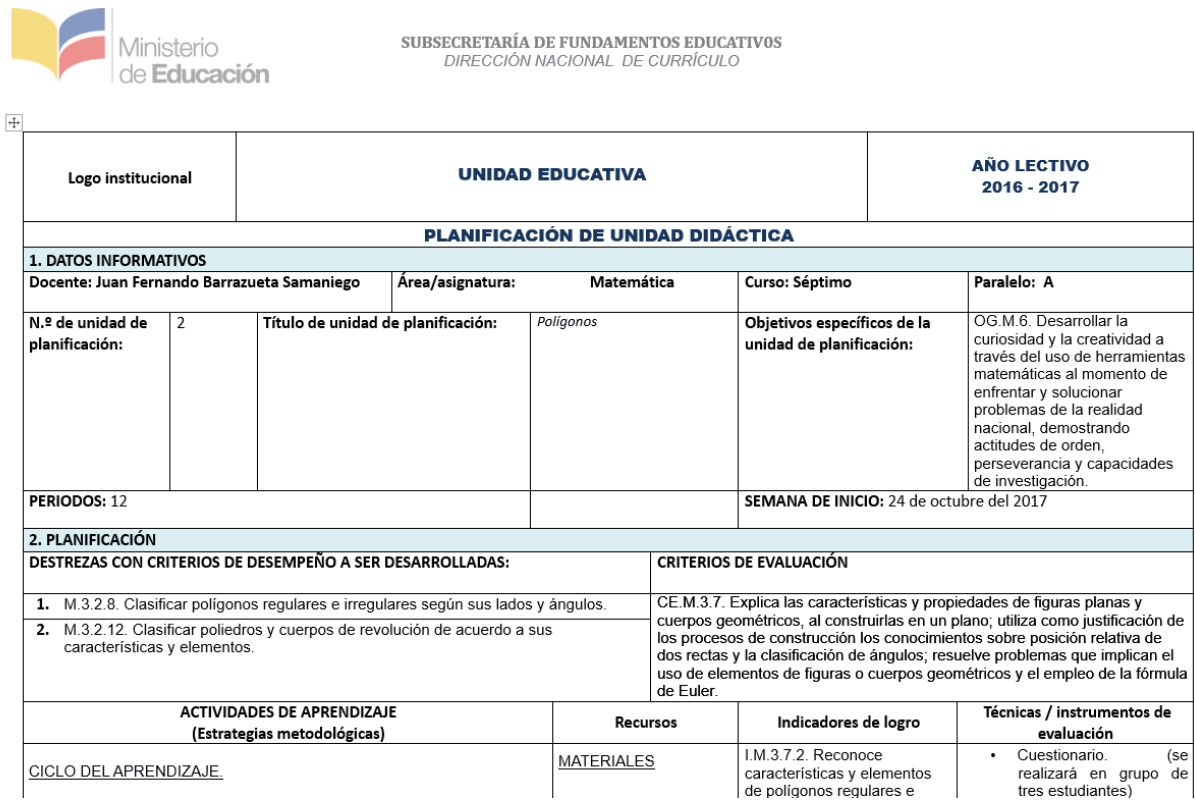

Ministerio

de Educación
SUBSECRETARÍA DE FUNDAMENTOS EDUCATIVOS DIRECCIÓN NACIONAL DE CURRÍCULO

\begin{tabular}{|c|c|c|c|}
\hline $\begin{array}{l}\text { Para todo este proceso se solicitará a los estudiantes trabajar en grupos de } \\
\text { tres. } \\
\text { RELACIÓN } \\
\text { Consiste en aprender en el contexto de las experiencias de la vida o del } \\
\text { conocimiento preexistente. Busca activar los conocimientos previos de los } \\
\text { estudiantes a traves de la recuperación de experiencias previas que hayan } \\
\text { tenido con respecto a los prismas y cilindros, para este caso en particular } \\
\text { se procederá a entregar a cada grupo, diferente material concreto que } \\
\text { representan cuatro tipos de prismas y el cilindro (el material concreto será } \\
\text { entregado al inicio de la clase), en la cual se identificará las partes o } \\
\text { elementos que lo conforman, específicamente como está estructurada. }\end{array}$ & $\begin{array}{l}\text { Internet } \\
\text { Textos: } \\
\text { Matemática } 7 \\
\text { Matemática } 8 \\
\text { Actualización y } \\
\text { Fortalecimiento } \\
\text { Curricular de la } \\
\text { Educación General } \\
\text { Básica } 2010 \\
\text { Geometría, } \\
\text { trigonometría, geometría } \\
\text { analítica } \\
\text { Matemáticas básicas y } \\
\text { geometría analítica } \\
\text { Fotocopias. } \\
\text { Marcadores. } \\
\text { Pizarra. } \\
\text { Cuaderno. } \\
\text { Lápices. } \\
\text { Regla. } \\
\text { Proyector. } \\
\text { Computador portátil. }\end{array}$ & $\begin{array}{l}\text { irregulares, poliedros y } \\
\text { cuerpos de revolución; los } \\
\text { relaciona con objetos del } \\
\text { entorno circundante; y aplica } \\
\text { estos conocimientos en la } \\
\text { resolución de situaciones } \\
\text { problema. (J.1., I.2.) }\end{array}$ & $\begin{array}{l}\text { 1. Identificar y describir } \\
\text { las caracteristicas } \\
\text { del cuel cuerpo } \\
\text { geométrico } \\
\text { entregado } \\
\text { 2. Encontrar en área y } \\
\text { el volumen del } \\
\text { cuerpo geométrico } \\
\text { que se entregará en } \\
\text { ese momento. }\end{array}$ \\
\hline
\end{tabular}




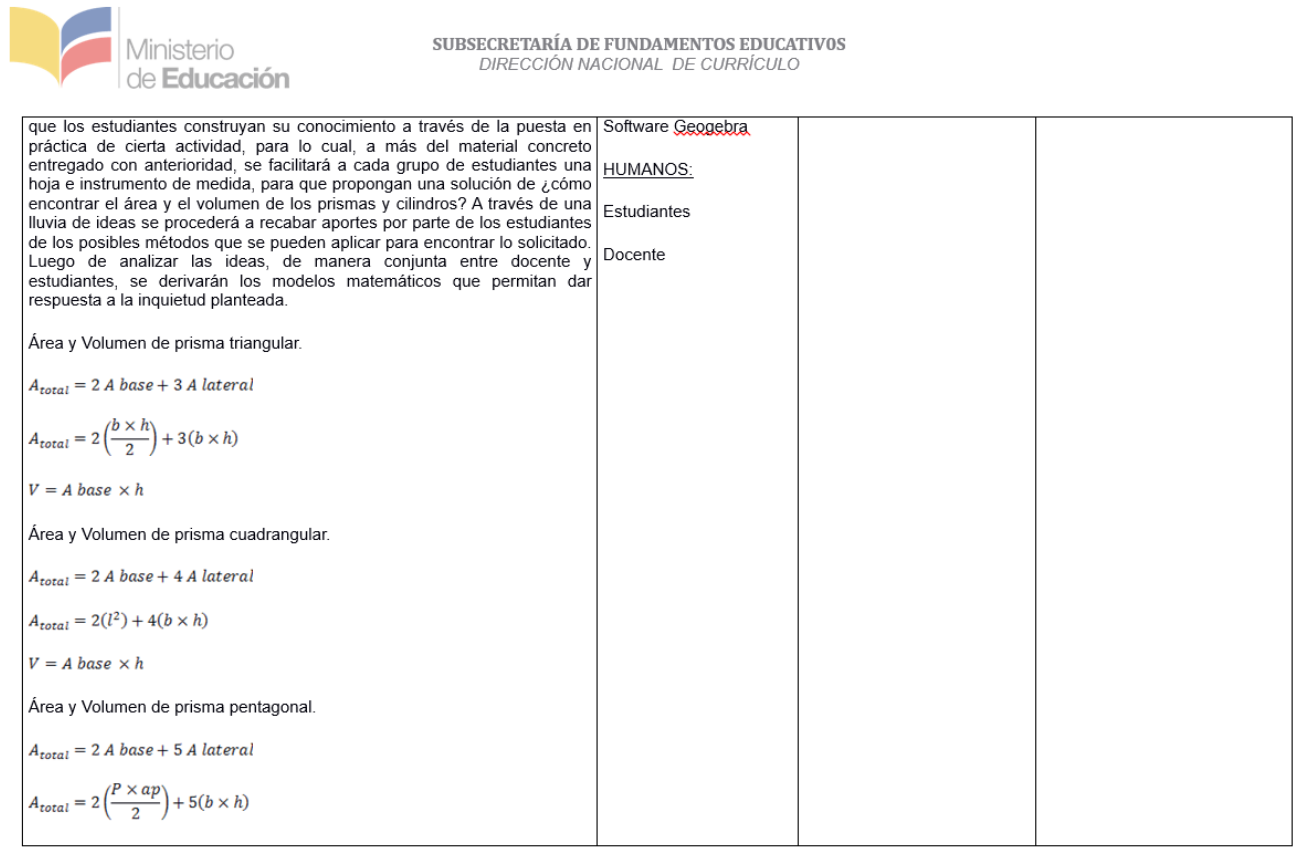

Ministerio

de Educación
SUBSECRETARÍA DE FUNDAMENTOS EDUCATIVOS DIRECCIÓN NACIONAL DE CURRICULO

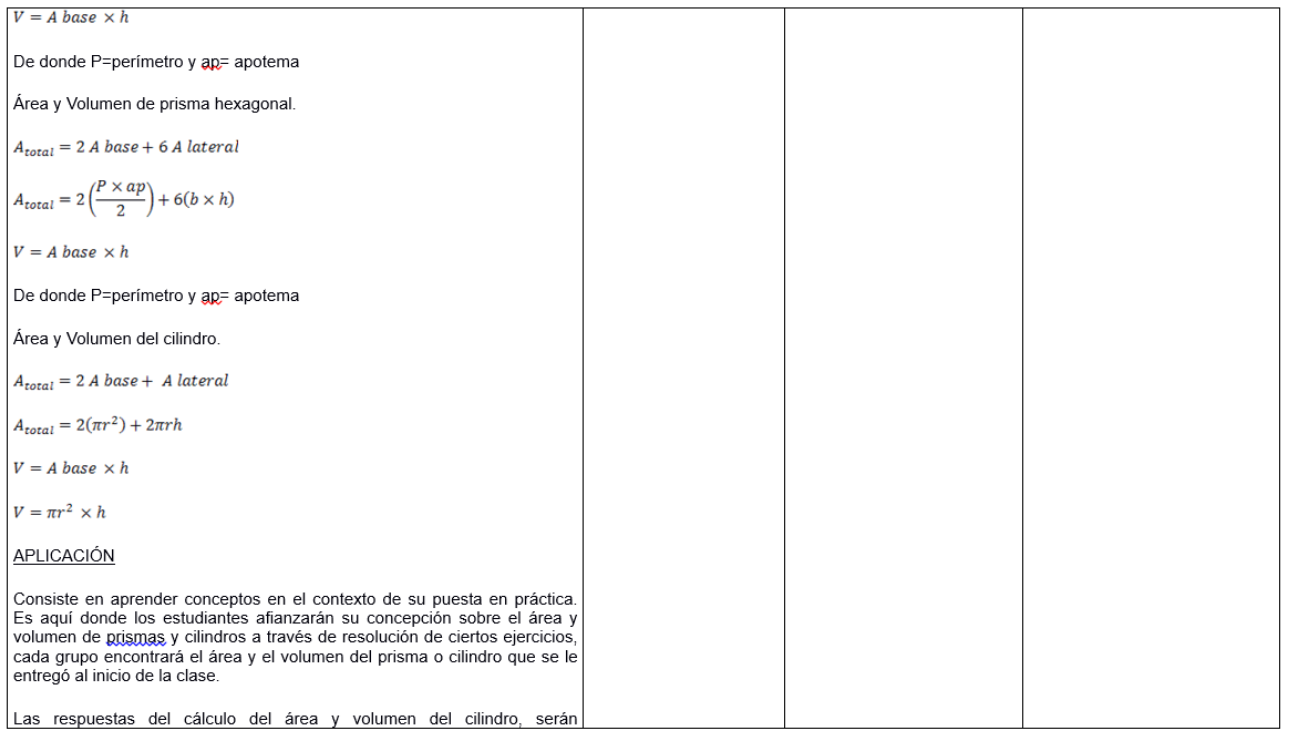




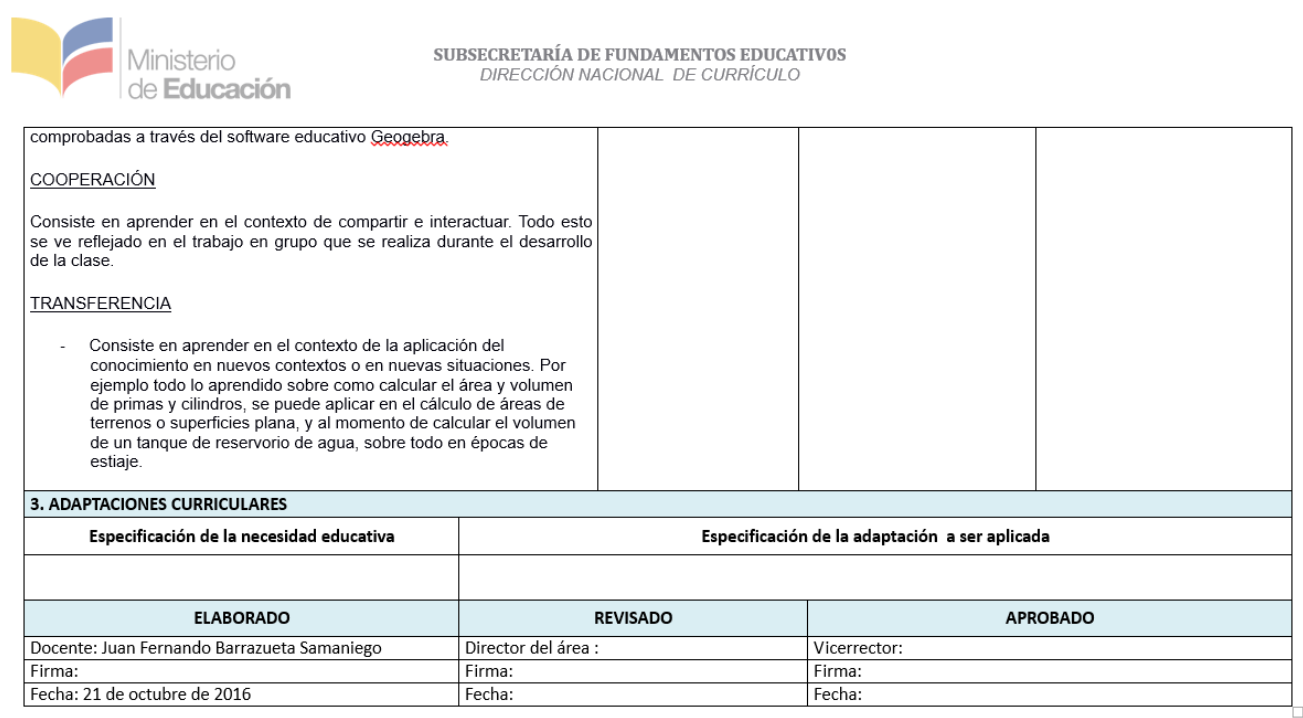

Figura 4. Modelo de planificación en el área de matemáticas.

Fuente. Propia. 